\title{
FUNCIONAMIENTO HIDROLÓGICO DE UNA PEQUEÑA CUENCA DE MONTAÑA CON MORFOLOGÍAS ACARCAVADAS EN EL PIRINEO CENTRAL
}

\author{
E. NADAL-ROMERO ${ }^{(1)}$, D. REGÜÉS ${ }^{(1)}$, J. LATRON $^{(2)}$, N. LANA-RENAULT ${ }^{(1)}$, \\ M. P. SERRANO-MUELA ${ }^{(1)}$ y C. MARTÍ-BONO ${ }^{(1)}$
}

(1) Instituto Pirenaico de Ecología, CSIC, Campus de Aula Dei. Avenida Montañana 1005, 50192. Zaragoza.

(2) Unidad de Ciencia del Suelo, Universidad de Girona, Campus de Montilivi, 17071. Girona correo electrónico de contacto: estelanr@ipe.csic.es

RESUMEN. La cuenca de Araguás fue monitorizada en 2004, con el objetivo de estudiar la dinámica de los procesos de meteorización y la dinámica geomorfológica e hidro-sedimentológica en una cuenca de montaña caracterizada por la presencia de morfologías acarcavadas. Este trabajo expone las principales características del funcionamiento hidrológico de la cuenca de Araguás, un espacio representativo de morfologías acarcavadas en la montaña media pirenaica. Entre octubre de 2005 y abril de 2007 se han registrado 79 crecidas. Los resultados obtenidos confirman la variabilidad de la respuesta hidrológica en medios acarcavados, así como su elevada capacidad de respuesta frente a cualquier precipitación. En general el coeficiente de escorrentía depende principalmente del volumen de precipitación y del caudal de base al inicio de la crecida. El pico de caudal presenta también buenas correlaciones con la intensidad de la precipitación. Igualmente, se observan diferencias estacionales muy importantes: en la estación seca la relación entre la intensidad de precipitación y el pico de caudal es significativa, mientras en la estación húmeda es inexistente, observándose mejores relaciones con la humedad antecedente. Finalmente, la forma del hidrograma sugiere dos tendencias hidrológicas diferentes en función de los mecanismos generadores de escorrentía y de su lugar de origen.

ABSTRACT. The Araguás catchment has been monitored since 2004 to study weathering, erosion and the hydrological and sediment response in order to understand the dynamics of a badland area in a relatively humid environment. This paper studies the main hydrological characteristics of the Araguás catchment, a representative badland area in a subhumid mountain environment. 79 floods have been recorded between October 2005 and April 2007. The results 
obtained confirm the temporal variability in the hydrological response within a small catchment with badland areas, and its high capacity of reaction against precipitation. Storm-flow mainly depends on the combination between rainfall volume and antecedent baseflow. On the other hand, a significant correlation was observed between maximum rainfall intensity and peak flow values. Moreover, seasonal differences were observed: during the dry season, significant correlations were observed between maximum rainfall intensity and peak flow; during the wet season this significant correlation disappeared and relationships with antecedent moisture conditions improved. Finally, the shape of the different hydrographs suggests two different hydrological patterns, due to runoff generation mechanisms.

Palabras clave: Respuesta hidrológica, variabilidad temporal, cárcavas, cuenca experimental, Pirineo Central

Key words: Hydrological response, temporal variability, badlands, experimental catchment, Central Pyrenees

Enviado el 15 de septiembre de 2008 Aceptado el 22 de octubre de 2009

\section{Introducción}

Según la Encyclopedia of Geomorphology (Goudie, 2004) los badlands son “paisajes erosivos profundamente disectados formados en rocas blandas, por lo general, en regiones semiáridas aunque no exclusivamente" (Harvey, 2004), ya que su presencia es común en medios subhúmedos de montaña (Regüés, 1995; Pardini, 1996; Torri y Rodolfi, 2000). Su origen es fluvial y se caracterizan por una densa red de drenaje que da lugar a un paisaje de microcuencas, con fuertes pendientes, en las que se desarrollan incisiones y profundos barrancos. En el Pirineo Central, su presencia está ligada a la presencia de margas eocenas (Solé Sabarís, 1942; Llopis, 1947) en un contexto de marcada estacionalidad climática.

En los últimos años, el equipamiento de cuencas experimentales ha permitido el estudio detallado de procesos hidrológicos y geomorfológicos en áreas acarcavadas en España. Sin embargo, buena parte de estos trabajos se han centrado en el análisis de la producción y transporte de sedimento y el estudio en detalle de los procesos erosivos (Clotet y Gallart, 1986; Cerdá y Navarro, 1997; Descroix y Olivry, 2002; Mathys et al., 2003; Regüés y Gallart, 2004; Desir y Marín, 2007; Martínez-Carreras et al., 2007). Otros trabajos, los menos, se han enfocado hacia problemas puramente hidrológicos (Solé et al., 1997; Gallart et al., 1998; Martínez-Mena et al., 1998; Cantón et al., 2001).

La cuenca de Araguás se ha monitorizado desde 2004 con el objetivo de estudiar los procesos de meteorización, erosión y la respuesta hidro-sedimentológica, y de esta manera comprender la dinámica hidro-geomorfológica de un área acarcavada en medios 
subhúmedos. Los resultados obtenidos hasta el momento han demostrado una gran variabilidad temporal del transporte de sedimento en suspensión, asociada a la dinámica estacional de los procesos de meteorización y erosión (Nadal-Romero et al., 2007, 2008b). Asimismo, se realizó un análisis preliminar de la variabilidad temporal de la respuesta hidrológica durante el año hidrológico 2005-2006 (Nadal-Romero et al., 2008a). En general, se observa una gran complejidad de las respuestas debido a la alta variabilidad temporal y espacial de las propiedades del regolito. Mathys et al. (2005) consideran que la dinámica del regolito, la intensidad de la lluvia y el volumen total de precipitación son los factores determinantes en la respuesta hidro-sedimentológica en zonas acarcavadas.

Los objetivos principales de este trabajo son: (i) estudiar el funcionamiento hidrológico de la cuenca de Araguás, (ii) definir la respuesta hidrológica frente a eventos pluviométricos de diferente magnitud e intensidad, y (iii) determinar los factores que mejor explican la respuesta hidrológica.

\section{2. Área de estudio}

La cuenca de Araguás $\left(0,45 \mathrm{~km}^{2}\right)$ es afluente por la margen derecha del río Lubierre, y se localiza en la zona central de la Depresión Interior Altoaragonesa (Pirineo Central) a $9 \mathrm{~km}$ de la ciudad de Jaca (Fig. 1). Su altitud máxima se alcanza a $1100 \mathrm{~m}$ s.n.m. y la más baja a 780 m s.n.m. La pendiente media se aproxima al $20 \%$ y las mayores pendientes (43\%) se localizan en laderas con exposición norte.

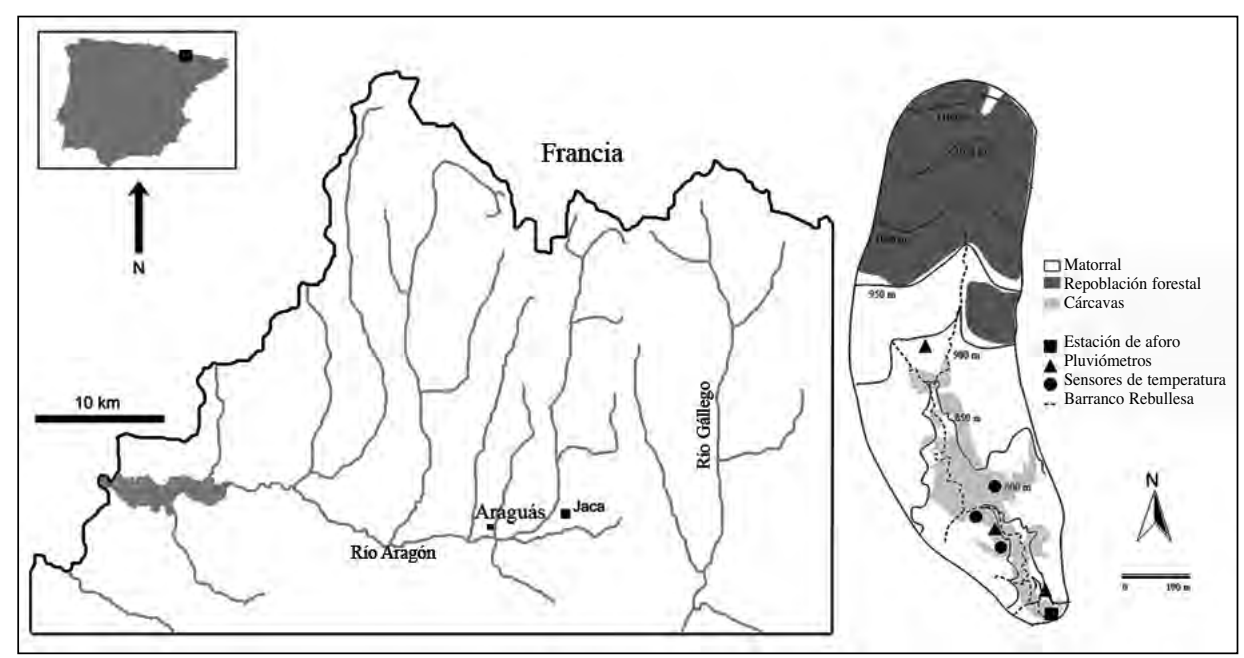

Figura 1. Localización e instrumentación de la cuenca de Araguás 
El sustrato geológico sobre el que se asienta está formado por dos grandes unidades: (i) en la parte superior predomina el flysch eoceno surpirenaico, constituido por una alternancia de areniscas carbonatadas y margas, afectado por pequeños deslizamientos que en algunas ocasiones pueden dar lugar a grandes movimientos en masa (Lorente et al., 2002); (ii) en la parte baja y media predomina la marga de Larrés, compuesta principalmente por minerales arcillosos (44\% de illita y clorita), carbonatos, con un elevado contenido de calcita y dolomita $(41 \%)$ y cuarzo (15\%). Además, se pueden observar pequeñas capas de areniscas y núcleos de concreciones carbonatadas. En relación a esta litología, se desarrolla una red de cárcavas (Fig. 2a), cuya presencia es mayor en exposición norte (Nadal-Romero et al., 2007).

El clima se define como submediterráneo de montaña, pero con cierta influencia continental y atlántica (Beguería et al., 2003), caracterizado por una marcada estacionalidad climática. La temperatura media anual oscila entre 9 y $10^{\circ} \mathrm{C}$, con una considerable amplitud térmica estacional y diaria. Son frecuentes los días de helada (80 días) principalmente en invierno, y esporádicamente en otoño y primavera. La precipitación media anual oscila entre 600 y $900 \mathrm{~mm}$, y se registra una media de 85 días de lluvia al año. Las precipitaciones tienden a concentrarse principalmente en otoño y primavera. En verano, son habituales las tormentas convectivas, de corta duración pero alta intensidad, y en

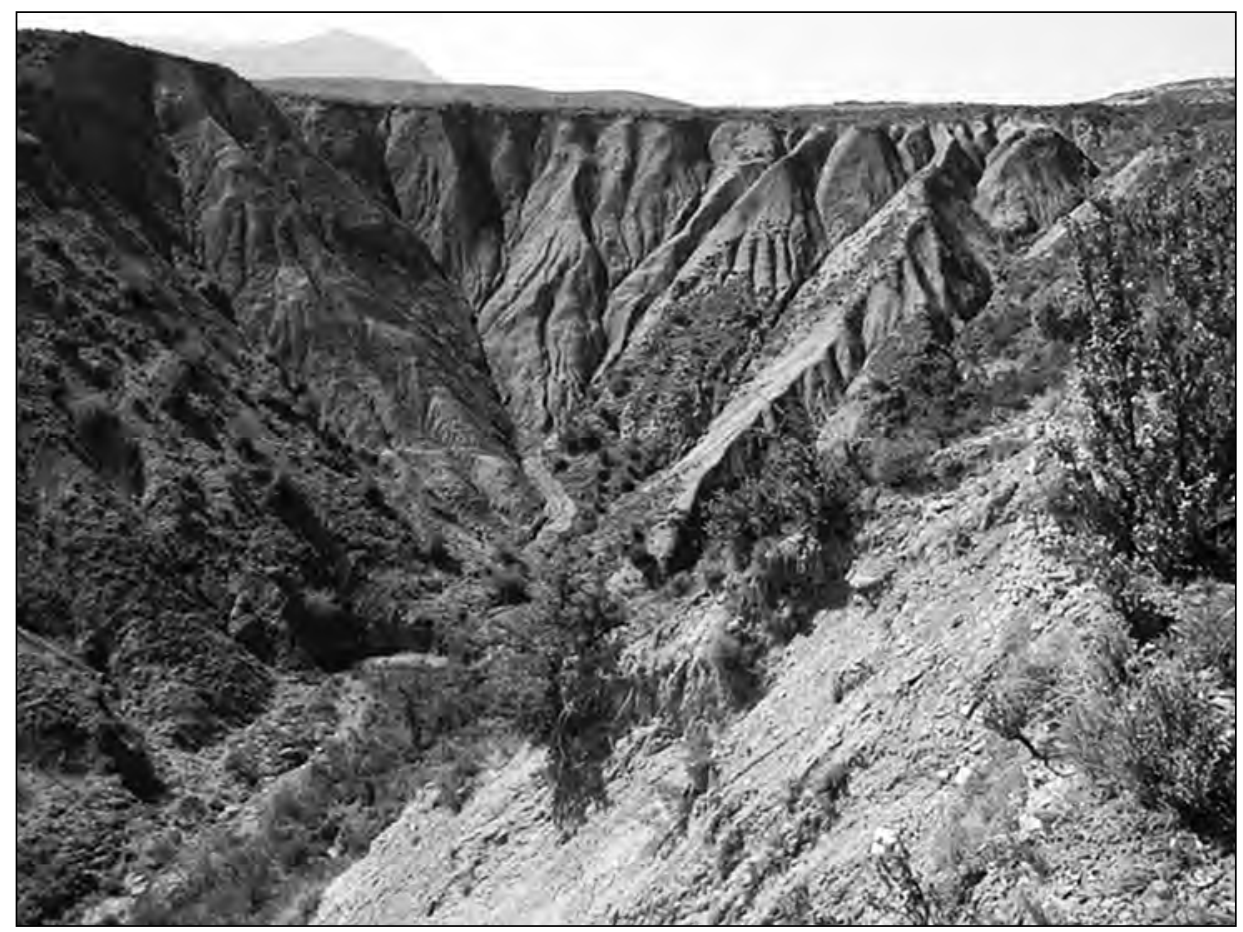

Figura 2a. Cárcavas en la cuenca de Araguás 
invierno el paso frecuente de frentes puede provocar precipitaciones en forma de nieve (Beguería y Vicente-Serrano, 2006).

En la cuenca de Araguás, se pueden observar diferentes ambientes, con un desarrollo de la vegetación muy heterogéneo y una distribución muy desigual: (i) en la parte alta de la cuenca se desarrolla un bosque de repoblación (llevada a cabo en las décadas de los sesenta y setenta) compuesto principalmente de pinos (Pinus sylvestris y Pinus nigra), ocupando un $30 \%$ de la superficie total (Fig. 2b); (ii) en la parte media, aparecen campos abandonados, en proceso de recolonización vegetal, junto con pastos y matorrales; y (iii) por último, en la zona acarcavada, predomina una vegetación de porte arbustivo y herbáceo (25\% del total de la cuenca) limitada por las condiciones extremas y el fuerte dinamismo que se sucede en las laderas (Fig. 2a).

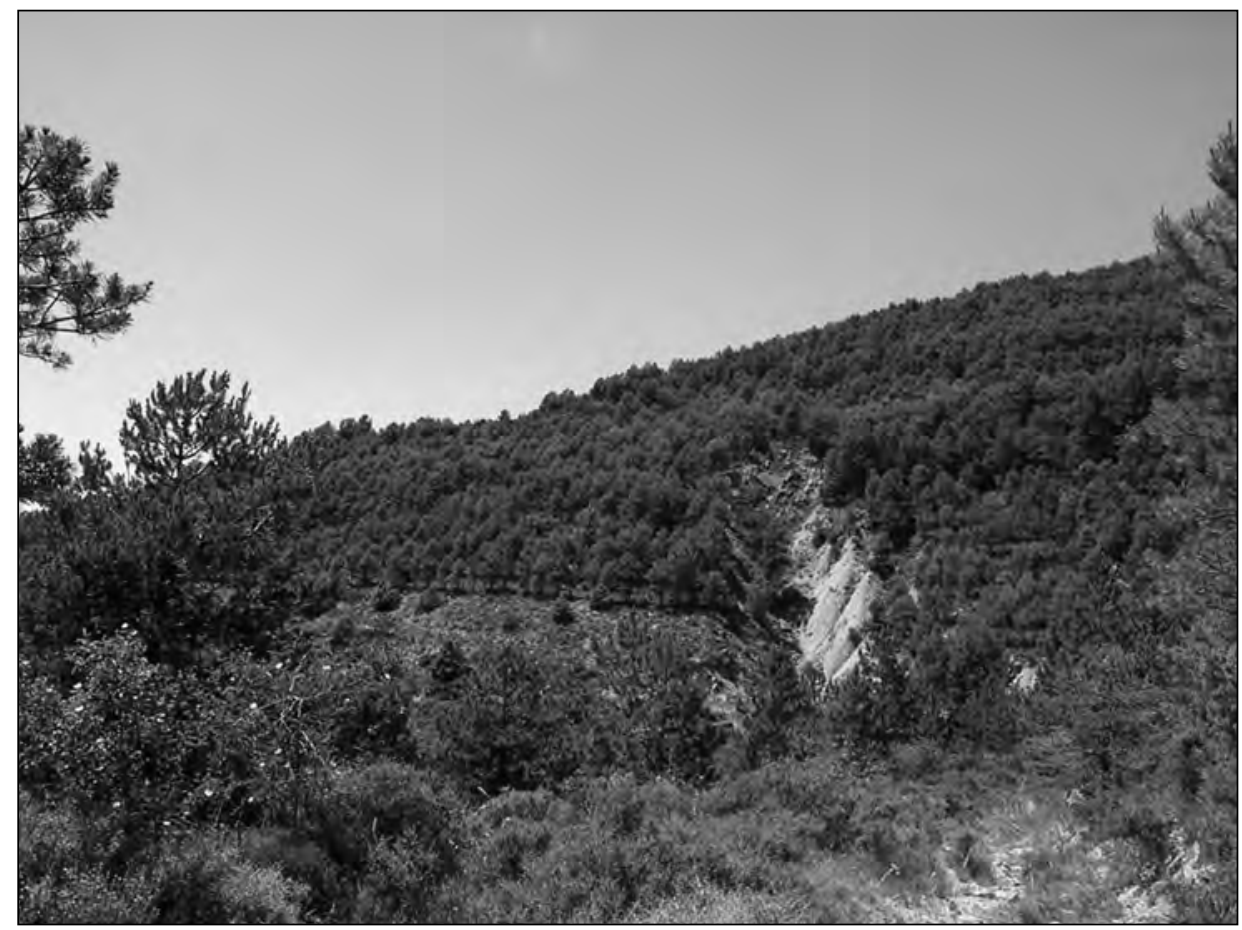

Figura 2b. Cuenca de Araguás, zona forestal vista desde el norte

\section{Equipamiento y métodos}

En el año 2005 se construyó una estación de aforo en la parte baja de la cuenca y se instaló el siguiente equipamiento para el estudio de la respuesta hidro-sedimentológica en la cuenca de Araguás: 
- Un sensor de ultrasonidos (Pepperl+Fuchs) y una sonda de presión (Keller DCX22) para la medición continua de la altura del agua.

- Un turbidímetro (Endress+Hauser) para la medición continua de la turbidez lo que permite, después de diferentes calibraciones (Nadal-Romero et al., 2008b), el cálculo de la concentración de sedimento en suspensión.

- Un tomamuestras automático (ISCO 3700), con capacidad para 24 botellas de medio litro cada una, programado para dispararse en el momento en que se produce un incremento en la altura o en la turbidez del agua. De este modo, se puede controlar el sedimento en suspensión y solución en momentos de crecida.

- Tres pluviómetros de balancín (Davis Instruments) conectados a un datalogger (Hobo), localizados a diferentes alturas (780, 800 y 1000 m s.n.m.).

Todos los instrumentos de medida, junto con un pluviómetro próximo a la estación de aforo, fueron conectados a un datalogger (Datataker DT-50) que registra la información cada 10 segundos, obteniendo valores medios cada 5 minutos.

Con los datos disponibles desde octubre de 2005 hasta abril de 2007 se han identificado 79 eventos. Un evento o crecida es definido como un incremento de caudal que supera en 1,5 veces el caudal de base inicial. Además, para cada evento el hidrograma fue dividido en dos componentes (delimitando de este modo la escorrentía de crecida), siguiendo el criterio utilizado por Hewlett y Hibbert (1967). Así, el hidrograma se divide por un línea recta con una pendiente constante, definida por Latron et al. (2008) como $1,831 \mathrm{~s} \mathrm{~s}^{-1} \mathrm{~km}^{-2}$ día $^{-1}$, y que se extiende desde el punto de inicio de la crecida hasta que intersecta con el tramo descendente del hidrograma.

Para cada crecida se han calculado los siguientes parámetros (Tabla 1a y 1b): precipitación total (Ptot, mm), intensidad máxima de precipitación (Imax, $\mathrm{mm} \mathrm{h}^{-1}$ ), escorrentía

Tabla 1a. Características generales de los eventos de crecida registrados en la cuenca de Araguás (28 de octubre de 2005 - 12 de abril de 2007) (Número de eventos $=79$ )

\begin{tabular}{|l|l|c|r|r|}
\hline & Mínima & Máxima & Media & Desv. estándar \\
\hline Ptot $(\mathrm{mm})$ & 0,8 & 49,8 & 10,26 & 9,33 \\
Imax. $\left(\mathrm{mm} \mathrm{h}^{-1}\right)$ & 1,2 & 62,4 & 13,14 & 12,71 \\
Esc. $(\mathrm{mm})$ & 0,01 & 19,07 & 1,71 & 3,61 \\
CE & 0,002 & 0,83 & 0,11 & 0,14 \\
Qmax. $\left(1 \mathrm{~s}^{-1}\right)$ & 4,44 & 2046,23 & 153,07 & 343,58 \\
Qb $\left(1 \mathrm{~s}^{-1}\right)$ & 0,04 & 70,56 & 6,60 & 10,74 \\
P1DA $(\mathrm{mm})$ & 0 & 25 & 5,65 & 6,46 \\
\hline
\end{tabular}

Ptot $=$ Precipitación total; Imax. = Máxima intensidad de precipitación en 5 minutos; Esc. $=$ Volumen de escorrentía; $\mathrm{CE}=$ Coeficiente de escorrentía; $\mathrm{Qmax} .=$ Pico de crecida; $\mathrm{Qb}=$ Caudal de base; $\mathrm{P} 1 \mathrm{DA}=$ Precipitación registrada un día anterior al evento. 


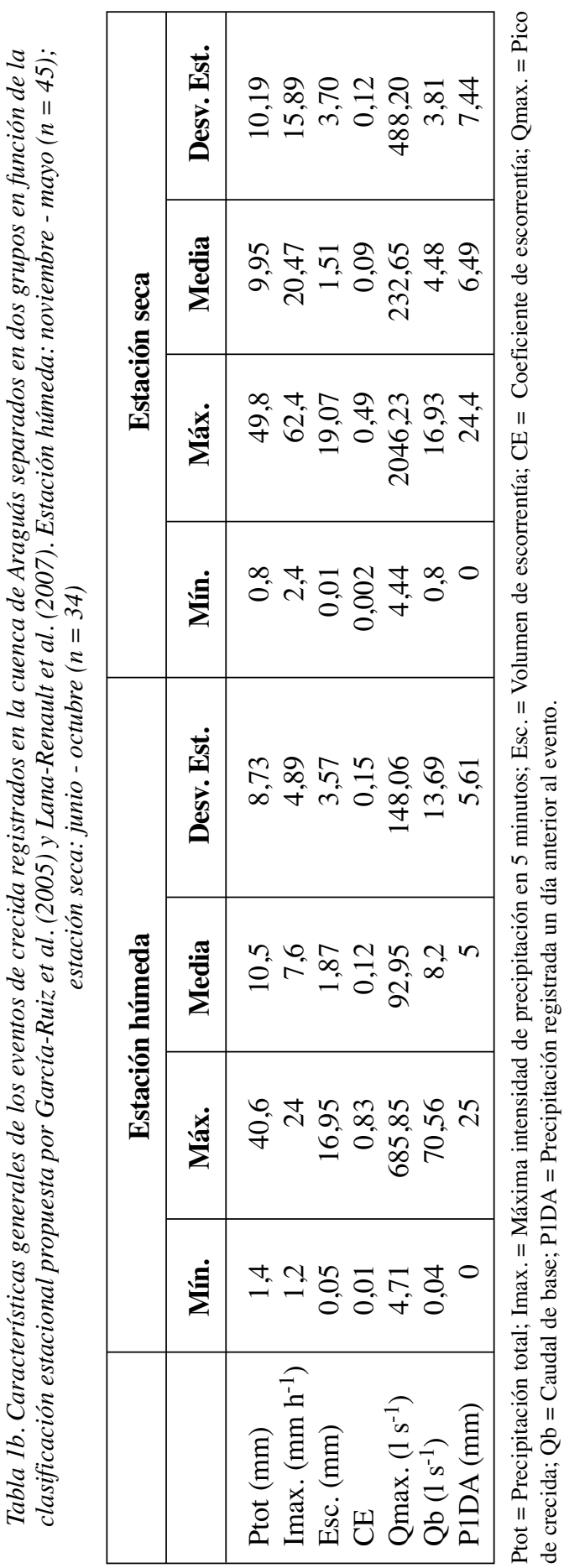


de crecida (Esc., mm), coeficiente de escorrentía (CE), pico de caudal (Qmax, $1 \mathrm{~s}^{-1}$ ), caudal de base $\left(\mathrm{Qb}, \mathrm{s}^{-1}\right)$ y la precipitación registrada el día anterior a cada evento (P1DA, mm).

Con la información disponible, se construyó una base de datos que permitió realizar diferentes análisis estadísticos, con el fin de investigar las relaciones estadísticas entre las diferentes variables.

\section{Resultados}

Durante el periodo de estudio (octubre de 2005 - abril de 2007) se han registrado 79 crecidas: 16 en primavera, 17 en verano, 28 en otoño y 18 en invierno. Siguiendo el esquema de clasificación propuesto por García-Ruiz et al. (2005) y Lana-Renault et al. (2007), 45 de las crecidas se produjeron durante la estación húmeda (noviembre-mayo) y 34 en la estación seca (junio-octubre) (Tabla 1a y 1b). De este modo, destaca el hecho de que la cuenca de Araguás tiene una capacidad de respuesta muy elevada y que las crecidas se pueden producir durante todo el año debido a la rápida respuesta de las morfologías acarcavadas.

(i) La mayoría de los eventos son de pequeña magnitud: el $67 \%$ de los eventos se produce con una precipitación inferior a $10 \mathrm{~mm}$ y sólo un $13 \%$ se produce con una precipitación superior a $20 \mathrm{~mm}$. La máxima precipitación registrada fue 49,8 mm con un periodo de retorno de 1,8 años según García-Ruiz et al. (2000).

(ii) La máxima intensidad de precipitación (en 5 minutos) varía entre $1,2 \mathrm{~mm} \mathrm{~h}^{-1}$ y $62,4 \mathrm{~mm} \mathrm{~h}^{-1}$. Sin embargo, sólo un $10 \%$ de los eventos alcanza una intensidad de precipitación superior a $30 \mathrm{~mm} \mathrm{~h}^{-1}$. Las intensidades más altas se registran durante la estación seca (Tabla 1b).

(iii) La escorrentía de crecida oscila entre 0,01 y 19,07 mm, registrándose los valores mínimos en verano y los máximos en invierno. El pico de caudal varía entre 4,44 y $2046 \mathrm{l} \mathrm{s}^{-1}$.

(iv) El coeficiente de escorrentía varía entre 0,002 y 0,83 (Tabla 1a).

En la Fig. 3 se ha representado la evolución temporal del coeficiente de escorrentía de las 79 crecidas registradas en la cuenca de Araguás. El coeficiente de escorrentía expresa la escorrentía en términos relativos a la precipitación, y es un buen indicador de la respuesta de la cuenca, independientemente de la magnitud del evento (Lana-Renault, 2008). Destaca sobre todo la elevada variabilidad de respuestas observadas a lo largo del año. El máximo coeficiente se registra en primavera, mientras en verano se producen coeficientes muy bajos, ya que sólo una parte de la cuenca (la zona acarcavada) contribuye a la formación de escorrentía (Fig. 3). En otoño e invierno la respuesta es moderada, pudiendo alcanzar valores mayores de 0,3, aunque la mayoría de los eventos están por debajo de 0,2 . 


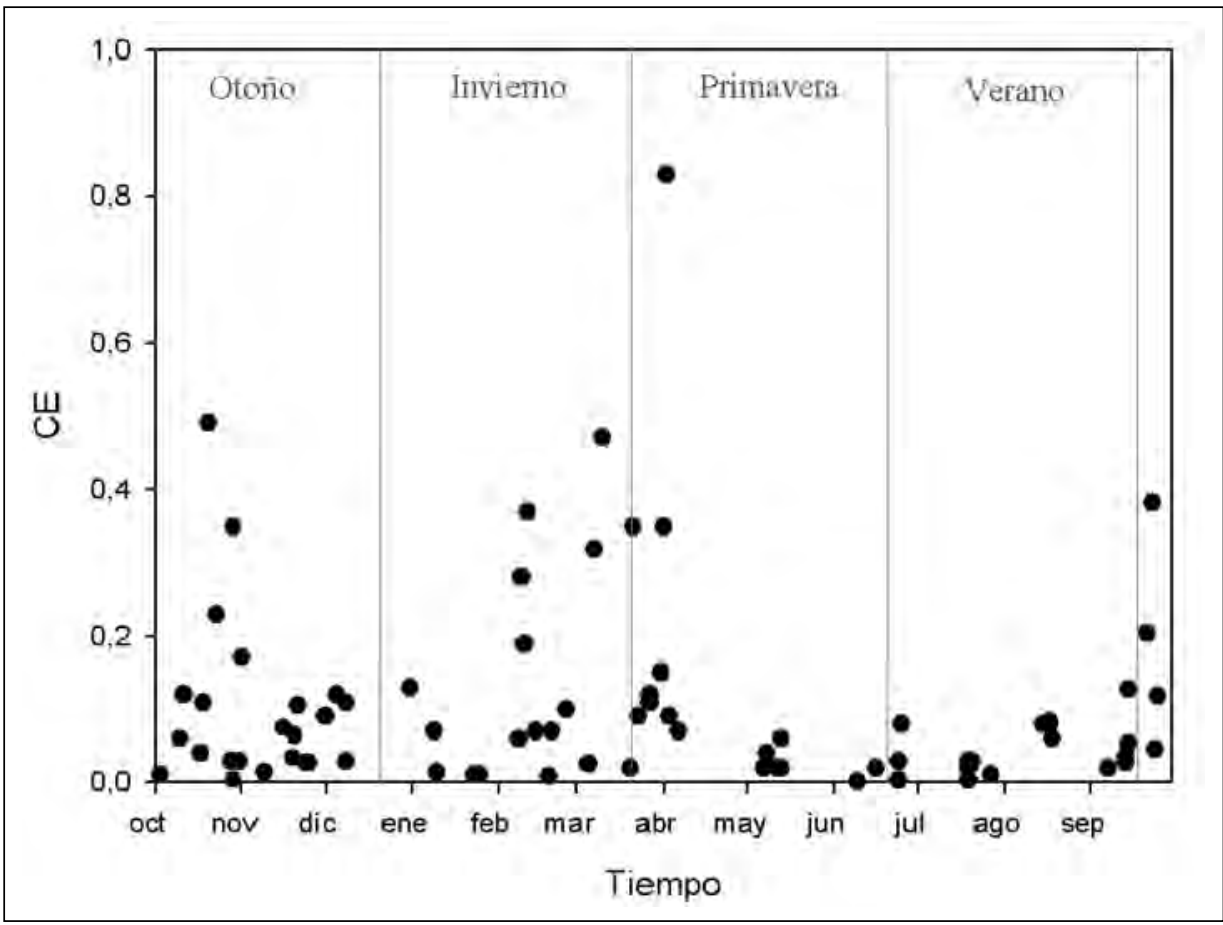

Figura 3. Evolución estacional del coeficiente de escorrentía (CE) de las 79 crecidas analizadas

La Fig. 4 ilustra la relación entre la precipitación y la escorrentía. Considerando el total de los eventos $(n=79)$ la relación es elevada $(\mathrm{R}=0,8519)$. No obstante, se pueden identificar dos tendencias: (i) Durante eventos con una precipitación débil $(\mathrm{P}<10 \mathrm{~mm})$ la escorrentía generada es siempre limitada, y sólo un evento supera los $3 \mathrm{~mm}$ de escorrentía; en ese caso, la relación entre las dos variables es muy baja $(n=50, \mathrm{R}=0,3052)$. (ii) Por el contrario, con precipitaciones superiores a $10 \mathrm{~mm}$ se observa un incremento progresivo de la escorrentía de crecida $(n=29, \mathrm{R}=0,9025)$. De este modo, la relación entre precipitación y escorrentía $(\mathrm{P}>10 \mathrm{~mm})$ es lineal y estadísticamente significativa ( $p<0,01)$ explicando el $90 \%$ de la varianza. Sin embargo, la transición entre las dos tendencias no está muy bien definida, como muestra la elevada dispersión de los puntos localizados entre 10 y $25 \mathrm{~mm}$ de precipitación. De hecho, una precipitación próxima a $25 \mathrm{~mm}$ puede producir una escorrentía entre 2 y $10 \mathrm{~mm}$. Este tipo de relación con dos tendencias ha sido ya observada en otras cuencas mediterráneas (Cosandey, 1993; Latron et al., 2008), aunque el límite establecido en este trabajo (10 mm) es más bajo que los determinados en los estudios anteriores.

La Fig. 5 presenta las relaciones entre el coeficiente de escorrentía, por un lado, y la precipitación total, la escorrentía de crecida, la máxima intensidad de precipitación y el caudal de base, por otro. 


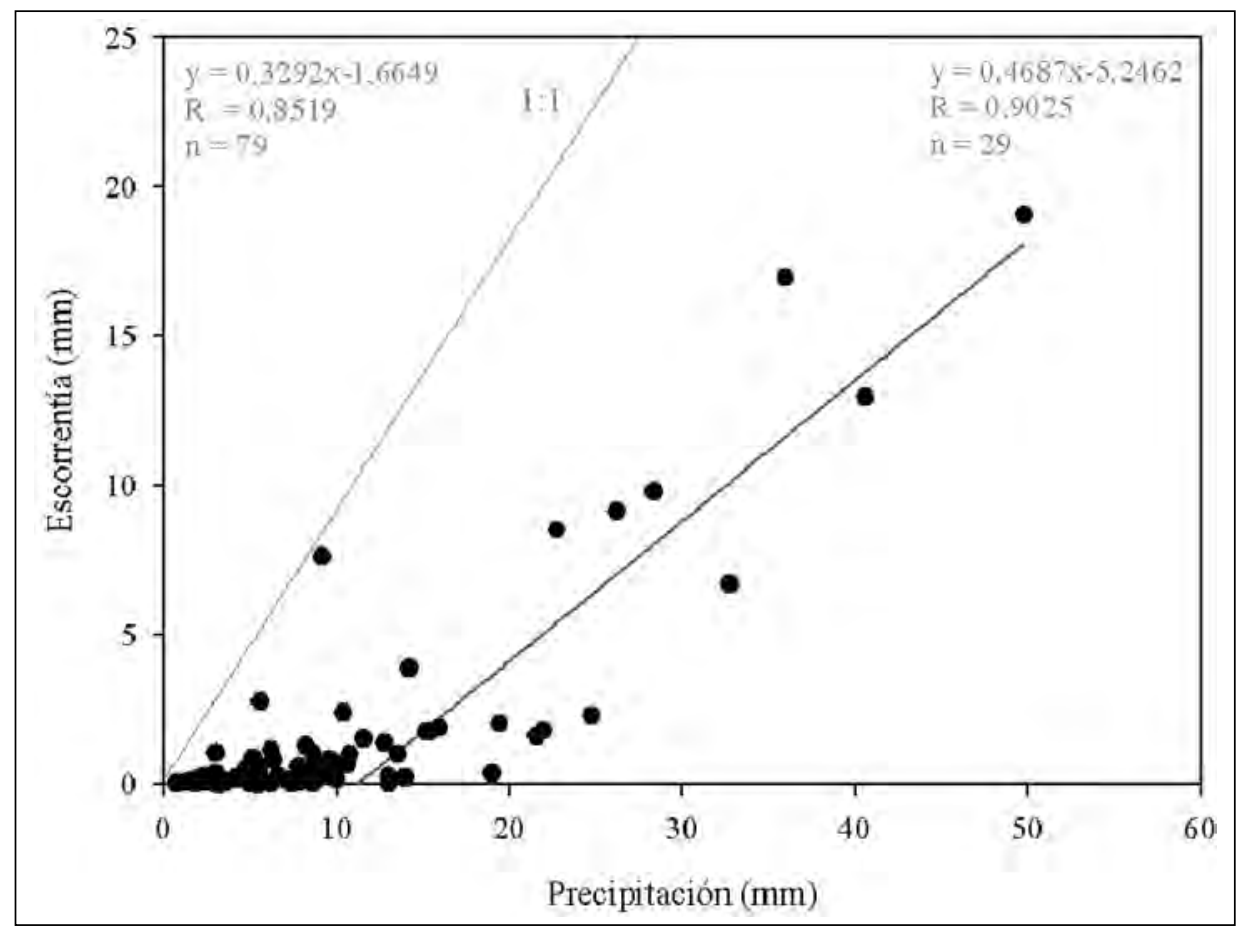

Figura 4. Relación entre la precipitación y la escorrentía de crecida en los eventos registrados en la cuenca de Araguás

La Fig. 5a revela que la precipitación total presenta una buena correlación con el coeficiente de escorrentía, significativa al nivel 0,01, explicando un $47 \%$ de la varianza $(\mathrm{R}=0,472)$. Sin embargo, la dispersión de la nube de puntos es evidente: una precipitación débil (por ej. 5,6 mm) puede generar un coeficiente de escorrentía muy diferente $(0,01$ y 0,49$)$; del mismo modo, un coeficiente de escorrentía elevado (próximo a 0,4 ) puede relacionarse con una precipitación de 3 y $50 \mathrm{~mm}$. En resumen, la variable precipitación no es suficiente para explicar la respuesta hidrológica en la cuenca de Araguás. Estas diferencias también han sido observadas en la cuenca de Arnás (Lana-Renault, 2008) y en la cuenca de Cal Rodó (Latron et al., 2008).

La relación entre la escorrentía de crecida y el coeficiente de escorrentía es muy elevada, explicando más del $70 \%$ de la varianza (nivel 0,01$)(\mathrm{R}=0,738)$. Sin embargo, en la Fig. 5b se observan dos tendencias claras: los coeficientes inferiores a 0,2 se producen siempre con valores de escorrentía muy bajos $(<3 \mathrm{~mm})$; y la dispersión es mayor para coeficientes elevados; de este modo, un coeficiente de escorrentía en torno a 0,35 puede ocurrir con escorrentías de crecidas que oscilan entre 1 y $20 \mathrm{~mm}$. Además, el máximo coeficiente de escorrentía $(0,83)$ no ha correspondido a la máxima escorrentía de crecida, sino a un valor moderado $(7,64 \mathrm{~mm})$. 


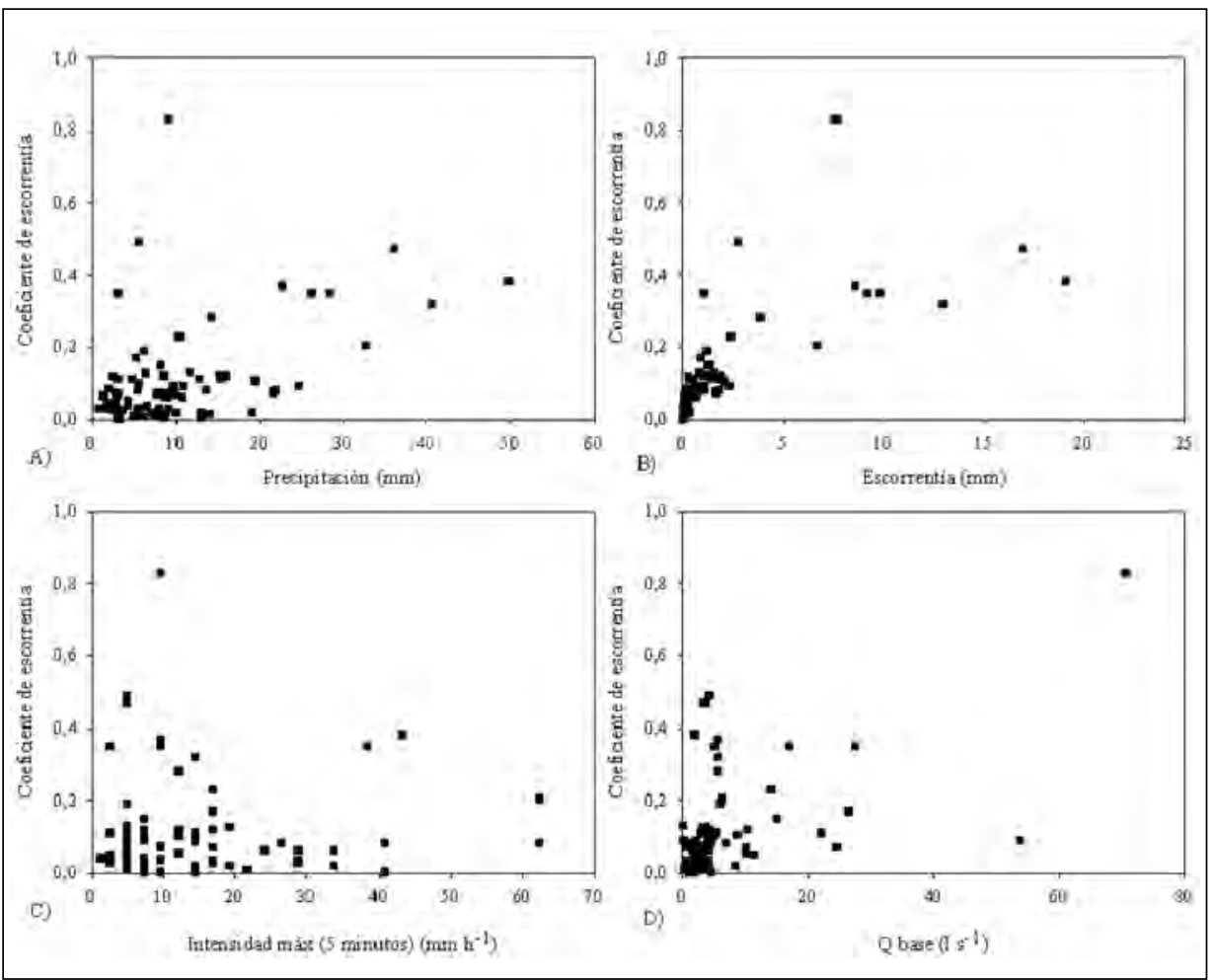

Figura 5. Relación entre el coeficiente de escorrentía y la precipitación (a), la escorrentía de crecida (b), la intensidad máxima de precipitación en 5 minutos (c) y el caudal de base específico previo a la crecida $(d)$

La Fig. 5c ilustra la relación entre la máxima intensidad de precipitación registrada durante 5 minutos y el coeficiente de escorrentía. Se debe destacar la fuerte heterogeneidad de la respuesta y la débil correlación estadística existente entre las dos variables $(\mathrm{R}=$ 0,068) (Tabla 2). El papel de la máxima intensidad de lluvia es difícil de explicar a escala de cuenca (Latron, 2003), pero en la cuenca de Araguás, la diversidad de ambientes contrastados (forestal, matorral y cárcavas) dificulta todavía más estas correlaciones. Trabajos previos exponen que en cuencas forestales el papel de la intensidad es muy bajo (Serrano et al., 2005, 2008) y, sin embargo, en zonas acarcavadas el papel de la intensidad en la respuesta hidrológica es esencial (Castelltort, 1995; Mathys et al., 2005).

Finalmente, la Fig. 5D presenta la relación entre el caudal de base (indicador de las condiciones de humedad antecedente de la cuenca) y el coeficiente de escorrentía. La relación estadística es buena y significativa al nivel $0,01(\mathrm{R}=0,536)$ (Tabla 1$)$. No obstante, la heterogeneidad es elevada y aunque el máximo coeficiente corresponde con el máximo caudal de base, no siempre se observan buenas relaciones. 
Tabla 2. Correlaciones lineales entre el coeficiente de escorrentía y el pico de caudal máximo $y$ diferentes variables para el total de las crecidas registradas $(n=79)$

\begin{tabular}{|l|c|c|}
\hline & Coeficiente de escorrentía & Pico de caudal $\left(\mathbf{l ~ s}^{-\mathbf{1}}\right)$ \\
\hline Ptot $(\mathrm{mm})$ & $\mathbf{0 , 4 7 2} * *$ & $\mathbf{0 , 6 3 7} * *$ \\
Imax. $\left(\mathrm{mm} \mathrm{h}^{-1}\right)$ & 0,068 & $\mathbf{0 , 6 2 9} * *$ \\
Esc. $(\mathrm{mm})$ & $\mathbf{0 , 7 3 8} * *$ & $\mathbf{0 , 6 4 7} * *$ \\
Qb $\left(1 \mathrm{~s}^{-1}\right)$ & $\mathbf{0 , 5 3 6 * *}$ & $\mathbf{0 , 2 5 0} *$ \\
P1DA $(\mathrm{mm})$ & 0,209 & 0,037 \\
\hline
\end{tabular}

Ptot $=$ Precipitación total; Imax. = Máxima intensidad de precipitación en 5 minutos; Esc. $=$ Volumen de escorrentía; $\mathrm{Qb}=$ Caudal de base; $\mathrm{P} 1 \mathrm{DA}=$ Precipitación registrada un día anterior al evento.

** Correlaciones significativas al nivel 0,01; * correlaciones significativas al nivel 0,05.

La Fig. 6 muestra la relación entre el pico de caudal y la máxima intensidad de precipitación (5 minutos), junto con el volumen de precipitación. Considerando todos los eventos, la relación es significativa (nivel 0,01 ) y explica cerca del $60 \%$ de la varianza. Los mayores picos de caudal están siempre asociados con eventos de gran volumen de precipitación y fuertes intensidades. Sin embargo, la respuesta es muy heterogénea y eventos con una intensidad de precipitación próxima a $40 \mathrm{~mm} \mathrm{~h}^{-1}$ pueden generar picos de caudal muy dispares: 4,5 y $20251 \mathrm{~s}^{-1}$.

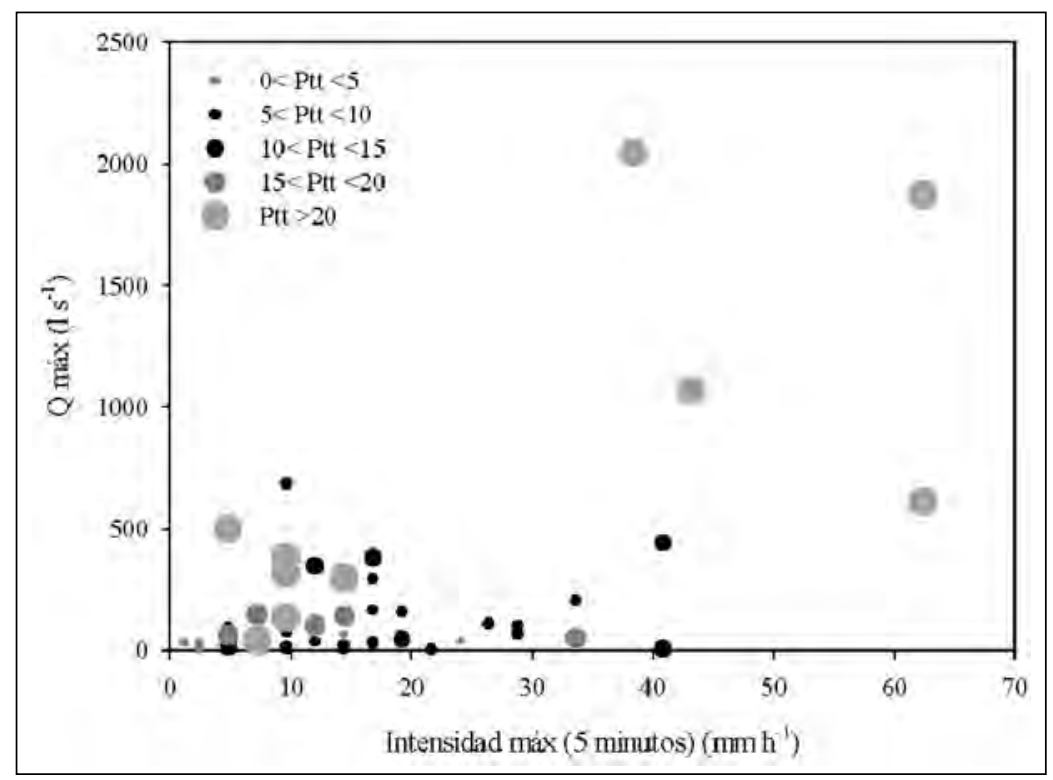

Figura 6. Relación entre la máxima intensidad de precipitación (5 minutos) y el pico de crecida para eventos de precipitación de diferente magnitud 
Con el objetivo de precisar las relaciones entre las diferentes variables se ha llevado a cabo un análisis de correlación; en primer lugar, se han utilizado todos los eventos (Tabla 2); en segundo lugar, se han diferenciado los eventos con precipitación inferior a $10 \mathrm{~mm}$ y superior a $10 \mathrm{~mm}$ (Fig. 4) (Tabla 3); por último, se ha realizado el análisis estadístico diferenciando los eventos en función de la clasificación estacional propuesta por García-Ruiz et al. (2005) y Lana Renault et al. (2007) (Tabla 4).

Tabla 3. Correlaciones lineales entre el coeficiente de escorrentía y el pico de caudal máximo y diferentes variables. El análisis de las correlaciones ha sido realizado separando las lluvias en función de la precipitación $(P<10 \mathrm{~mm}, n=53 ; P>10 \mathrm{~mm}, n=26)$

\begin{tabular}{|l|c|c|c|c|}
\hline & \multicolumn{2}{|c|}{ P<10 mm } & \multicolumn{2}{c|}{ P>10 mm } \\
\hline & $\begin{array}{c}\text { Coeficiente } \\
\text { de escorrentía }\end{array}$ & $\begin{array}{c}\text { Pico de } \\
\text { caudal }\left(\mathbf{l ~ s}^{-1}\right)\end{array}$ & $\begin{array}{c}\text { Coeficiente } \\
\text { de escorrentía }\end{array}$ & $\begin{array}{c}\text { Pico de } \\
\text { caudal }(\mathbf{l ~ s} \text {-1) }\end{array}$ \\
\hline Ptot $(\mathrm{mm})$ & 0,152 & $0,306^{*}$ & $\mathbf{0 , 7 0 3} * *$ & $\mathbf{0 , 5 7 3} * *$ \\
Imax. $\left.(\mathrm{mm} \mathrm{h})^{-1}\right)$ & $-0,070$ & $\mathbf{0 , 2 8 2} * *$ & 0,017 & $\mathbf{0 , 6 5 3} * *$ \\
Esc. $(\mathrm{mm})$ & $\mathbf{0 , 9 4 7 * *}$ & $\mathbf{0 , 8 5 8} * *$ & $\mathbf{0 , 9 0 4} * *$ & $\mathbf{0 , 5 3 7 * *}$ \\
Qb $\left(1 \mathrm{~s} \mathrm{~s}^{-1}\right)$ & $\mathbf{0 , 6 4 6}^{* *}$ & $\mathbf{0 , 7 6 5} * *$ & 0,327 & 0,252 \\
P1DA $(\mathrm{mm})$ & $0,341^{*}$ & $\mathbf{0 , 4 1 6} * *$ & 0,301 & 0,282 \\
\hline
\end{tabular}

** Correlaciones significativas al nivel 0,01; * correlaciones significativas al nivel 0,05.

Tabla 4. Correlaciones lineales entre el coeficiente de escorrentía y el pico de caudal máximo $y$ diferentes variables. El análisis de las correlaciones ha sido realizado separando los eventos en función de la clasificación estacional propuesta por García Ruiz et al.(2005) y Lana Renault et al. (2007) (estación húmeda, $n=45$; estación seca $n=34$ )

\begin{tabular}{|c|c|c|c|c|}
\hline & \multicolumn{2}{|c|}{ Estación húmeda } & \multicolumn{2}{|c|}{ Estación seca } \\
\hline & $\begin{array}{c}\text { Coeficiente } \\
\text { de escorrentía }\end{array}$ & $\begin{array}{c}\text { Pico de } \\
\text { caudal }\left(1 \mathbf{s}^{-1}\right)\end{array}$ & $\begin{array}{c}\text { Coeficiente } \\
\text { de escorrentía }\end{array}$ & $\begin{array}{c}\text { Pico de } \\
\text { caudal }\left(1 \mathrm{~s}^{-1}\right)\end{array}$ \\
\hline Ptot (mm) & $0,437 * *$ & $\mathbf{0 , 5 6 0} * *$ & $\mathbf{0 , 5 5 5} * *$ & $\mathbf{0 , 7 8 5} * *$ \\
\hline $\operatorname{Imax} .\left(\mathrm{mm} \mathrm{h}^{-1}\right)$ & 0,108 & 0,261 & 0,237 & $0,668 * *$ \\
\hline Esc. (mm) & $\mathbf{0 , 7 5 7} * *$ & $\mathbf{0 , 8 1 6 * *}$ & $\mathbf{0 , 7 3 1} * *$ & $\mathbf{0 , 7 4 7} * *$ \\
\hline $\mathrm{Qb}\left(1 \mathrm{~s}^{-1}\right)$ & $\mathbf{0 , 5 8 1} * *$ & $\mathbf{0 , 5 8 5} * *$ & $0,410^{*}$ & $\mathbf{0 , 4 8 6}{ }^{* *}$ \\
\hline P1DA (mm) & $\mathbf{0 , 4 6 0} * *$ & $0,343 *$ & $-0,067$ & $-0,084$ \\
\hline
\end{tabular}

** Correlaciones significativas al nivel 0,01; * correlaciones significativas al nivel 0,05. 
La correlación entre el coeficiente de escorrentía (CE) y la precipitación total (Ptot) es significativa en todos los casos de análisis (nivel 0,01), exceptuando los eventos con una precipitación inferior a $10 \mathrm{~mm}$. Sin embargo, la relación entre el coeficiente de escorrentía y la intensidad máxima de precipitación en 5 minutos (Imax) es inexistente, a pesar de la importante presencia de áreas acarcavadas en la zona baja de la cuenca de Araguás.

Diferentes resultados se obtienen de las relaciones entre el coeficiente de escorrentía y el caudal de base $(\mathrm{Qb})$. Se observan relaciones significativas $(0,01)$ con la totalidad de los eventos, los eventos de precipitación inferior a $10 \mathrm{~mm}$ y los eventos de estación húmeda; los eventos de estación seca presentan relaciones estadísticamente significativas al nivel 0,05; y por último, no existe relación con los eventos de precipitación superior a $10 \mathrm{~mm}$.

Finalmente, las relaciones entre el coeficiente de escorrentía y la precipitación antecedente un día antes del evento (P1DA) sólo son significativas (nivel 0,05) durante los eventos de precipitación inferiores a $10 \mathrm{~mm}$.

Por su parte el pico de caudal (Qmax) se correlaciona con la precipitación total y con la escorrentía de crecida en todos los casos de estudio. La intensidad máxima de precipitación y el pico de caudal se correlacionan también en todos los análisis (nivel 0,01), excepto en los eventos registrados durante la estación húmeda. Sorprendentemente las correlaciones más elevadas se observan durante la estación seca (junio-octubre), hecho que puede estar relacionado con la presencia de zonas acarcavadas ( $25 \%$ de la cuenca) y por la elevada frecuencia de tormentas convectivas de gran intensidad.

Por último, sólo existen correlaciones significativas entre el pico de caudal y la precipitación antecedente si se consideran los eventos con precipitación inferior a $10 \mathrm{~mm}$ y durante los eventos de estación seca.

Recapitulando todos los resultados estadísticos, reflejados en las Tablas 2, 3 y 4, destacan los puntos siguientes:

i. Los eventos con baja precipitación $(\mathrm{p}<10 \mathrm{~mm}$ ) están condicionados principalmente por las condiciones de humedad de la cuenca (Qb y P1DA) (Tabla 3).

ii. Los eventos con precipitación elevada ( $p>10 \mathrm{~mm}$ ) están limitados especialmente por las características de la precipitación (Ptot e Imax) (Tabla 3).

iii. Los eventos de estación húmeda están condicionados por las condiciones de humedad previa y la precipitación total. Sin embargo, no se observan buenas relaciones con la máxima intensidad de precipitación (Tabla 4).

iv. Los eventos de estación seca están condicionados por las características de la precipitación (Ptot e Imax) y el caudal de base, pero no por la precipitación antecedente registrada un día antes del evento (Tabla 4).

La Fig. 7 presenta 4 hidrogramas con sus correspondientes hietogramas. Los dos primeros eventos se produjeron durante la estación seca (junio-octubre). Las formas de los 
hidrogramas son muy similares, aunque los eventos son de diferente magnitud. La respuesta hidrológica en ambos casos, está caracterizada por rápidas respuestas (en torno a 30 minutos). Asimismo, se observa un rápido ascenso y descenso de la curva del hidrograma (Fig. 7a y $7 b$ ), que reproduce con bastante fidelidad la forma del hietograma. Esto sugiere que al menos una parte importante de la cuenca funciona por flujo hortoniano, es decir, que la capacidad de infiltración es inferior a la intensidad de la precipitación, generándose escorrentía sin necesidad de que el suelo se haya saturado. De hecho, la forma tan sencilla y repetitiva de los hidrogramas refleja el predominio de la escorrentía hortoniana, sin que afloren otros mecanismos internos en la cuenca que puedan variar la velocidad e intensidad de la respuesta.
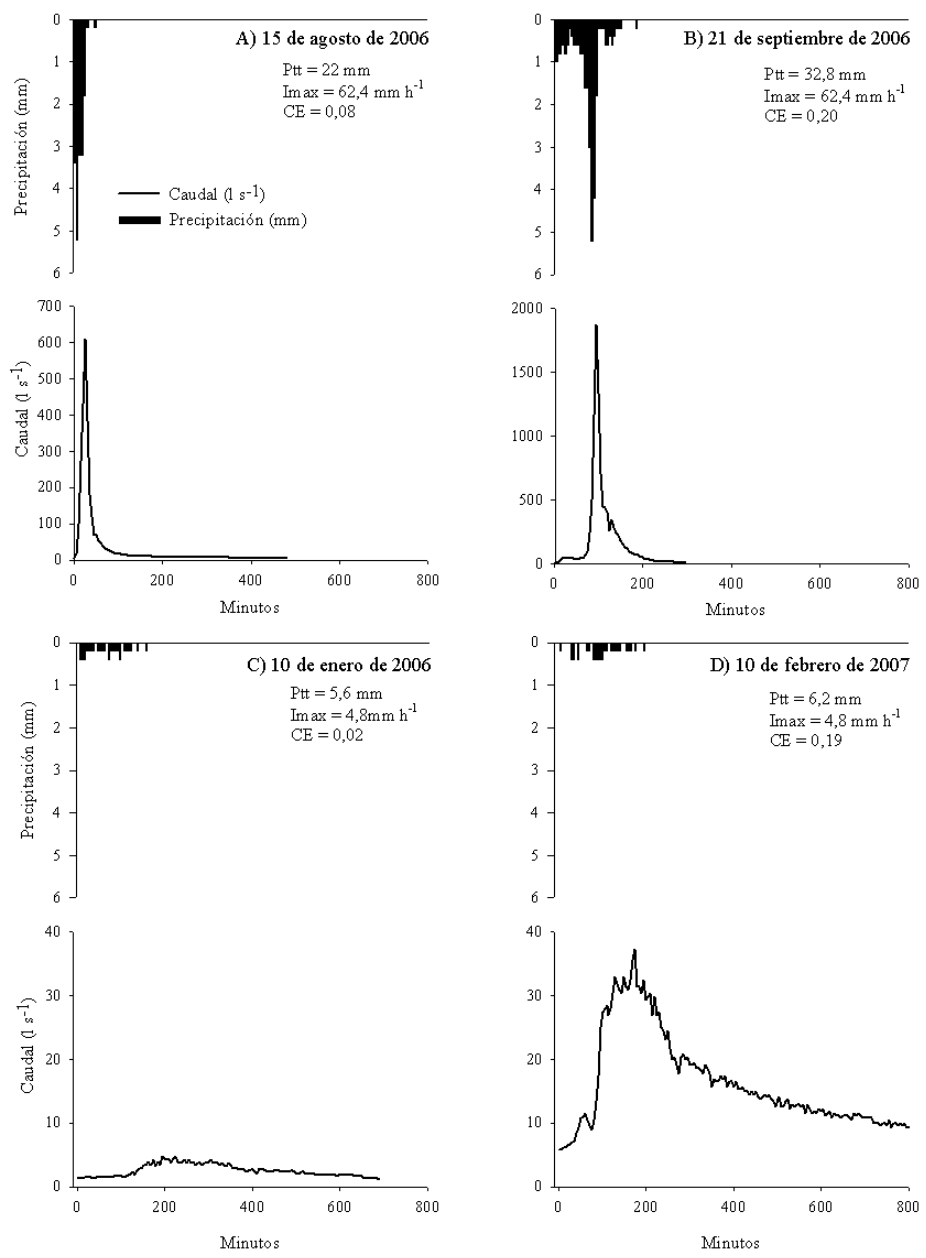

Figura 7. Hidrogramas y hietogramas para 4 eventos de magnitud contrastada en la cuenca de Araguás. Los eventos $A$ y $B$ se registraron durante la estación seca, y los eventos $C$ y $D$ durante la estación húmeda. Nótese que la escala en el eje de ordenadas varía en cada caso 
Sin embargo, hay momentos en los que se refleja la influencia de la zona forestal de la cuenca en la forma y características del hidrograma (Fig. 7c y 7d). Los dos eventos representados en las Fig. 7c y 7d se produjeron durante la estación húmeda y se caracterizan por unos valores de intensidad y de precipitación bajos. La forma del hidrograma es muy significativa: se observa un caudal de base moderado al inicio del evento y lentamente se produce un incremento de caudal (más acusado en el evento representado en la Fig. 7d). Cuando se alcanza el pico de caudal, éste puede mantenerse estable durante un tiempo, para después producirse una lenta y progresiva tendencia decreciente (Fig. 7c y 7d). Estos cambios sugieren un cambio en el origen y en los mecanismos de escorrentía activos en la cuenca de Araguás. De ahí puede deducirse que durante ciertos eventos en la cuenca de Araguás, principalmente durante la estación húmeda, un porcentaje desconocido de la escorrentía se origina en la zona forestal. En cambio, se puede afirmar que durante la estación seca la escorrentía se produce esencialmente en la zona acarcavada. Por esta razón, como se ha visto anteriormente, la correlación entre la intensidad de precipitación y el pico de caudal es muy superior durante la estación seca.

\section{Discusión y conclusiones}

Esta investigación presenta la variabilidad temporal de la respuesta hidrológica en una pequeña cuenca de montaña, caracterizada por la importante presencia de cárcavas. Resultados similares han sido observados en trabajos previos en cuencas de similares características (Scoging, 1982; Cerdá, 1999; Cantón et al., 2001; Mathys et al., 2003).

La relación entre la precipitación y la escorrentía de crecida es elevada, aunque se pueden diferenciar dos tendencias claras (Fig. 4): respuestas limitadas ante eventos con una precipitación inferior a $10 \mathrm{~mm}$ y respuestas en progresivo aumento para eventos con una precipitación mayor de $10 \mathrm{~mm}$. Este doble comportamiento se observa en otras cuencas mediterráneas (Cosandey, 1993; Latron, 2003; Latron et al., 2008), aunque el límite de ruptura establecido en Araguás $(10 \mathrm{~mm})$ es más bajo que los determinados en los estudios anteriores. Del mismo modo, Jordan (1992 y 1994) diferenció dos tendencias en la relación entre precipitación y escorrentía, en cuencas suizas, con un clima más templado, aunque el umbral de repuesta es muy pequeño (unos pocos mm) y la dispersión de los puntos es menor.

Los datos acumulados desde octubre de 2005 permiten realizar una primera caracterización del funcionamiento hidrológico en la cuenca de Araguás. En primer lugar destaca su alta capacidad de respuesta. Así, durante el año hidrológico 2005-2006 en la cuenca de Araguás se registraron 44 crecidas, mientras en las cuencas de Arnás y San Salvador (localizadas a menos de $10 \mathrm{~km}$ de distancia pero con mayor cubierta vegetal) se registraron 12 y 6 eventos respectivamente (García-Ruiz et al., 2008).

En segundo lugar, la cuenca reacciona de forma súbita, con tiempos de respuesta inferiores a una hora. Resultados similares se han observado en estudios hidrológicos en 
otras zonas acarcavadas (Imeson et al., 1982; Calvo et al., 1991; Solé-Benet et al., 1997), debido a su baja capacidad de infiltración (Regüés y Gallart, 2004). Por ello, en muchos casos se han identificado como la única fuente de escorrentía en respuesta a pequeños eventos de fuerte intensidad (Latron y Gallart, 1995). En general, en la cuenca de Araguás se observan bajos coeficientes de escorrentía (en la mayoría de los eventos el coeficiente es inferior a 0,2 ), lo que probablemente refleja la contribución exclusiva de la zona acarcavada. En estas condiciones, la parte forestal contribuye de manera limitada, confirmando que un aumento en la cubierta vegetal controlaría el volumen de escorrentía (Beguería et al., 2003; López-Moreno et al., 2006).

En conclusión, en la cuenca de Araguás se pueden distinguir dos comportamientos hidrológicos muy diferentes, que contribuyen a explicar la variabilidad y complejidad de la respuesta frente a eventos pluviométricos:

i. En la parte alta de la cuenca, donde predomina el ambiente forestal (29\% del total de la cuenca), el funcionamiento hidrológico es muy moderado, debido tanto a la interceptación como a la mayor capacidad de infiltración de los suelos. Este es un comportamiento hidrológico semejante al observado en la cuenca de San Salvador (Serrano et al., 2005, 2008). Muy probablemente, los mecanismos de generación de escorrentía funcionan por procesos de saturación.

ii. En la zona acarcavada, los mecanismos de generación de escorrentía son muy rápidos, relacionados con la baja permeabilidad del regolito, de manera que el aporte de agua desde las laderas al cauce se debe a que, en general, la intensidad de la precipitación supera a la capacidad de infiltración (flujo hortoniano).

\section{Agradecimientos}

Este trabajo se ha realizado con el apoyo de los siguientes proyectos: "Caracterización y modelización de procesos y regímenes hidrológicos en cuencas aforadas para la predicción en cuencas no aforadas" (CANOA, CGL 2004-04919-C02-01), "Procesos y balances de sedimentos a diferentes escalas espaciales en ambientes mediterráneos: Efectos de las fluctuaciones climáticas y los cambios de uso del suelo" (PROBASE, CGL2006-11619/HID) y "Comportamiento y modelización espacio temporal de la transferencia de sedimentos en distintos usos del suelo" (CETSUS, CGL2007-66644C04-01/HID) todos financiados por la CICYT, y "Validación de un modelo acoplado de simulación de procesos hidrológicos e hidráulicos utilizando datos de cuencas experimentales en montaña mediterránea" (PM088/2006), financiado por la Diputación General de Aragón (DGA). El mantenimiento y mejora de la cuenca se ha llevado a cabo mediante el Convenio CSIC/Ministerio de Agricultura (Dirección General de Conservación de la Naturaleza) - "Red de estaciones Experimentales de seguimiento y evaluación de la erosión y desertificación" (RESEL), financiado por el Ministerio de Agricultura. El primer autor contó con una beca predoctoral I3P concedida por el CSIC en el contexto del Programa Social Europeo. 


\section{Referencias bibliográficas}

BEgUeríA, S., ViCENTE-SERRANO, S.M. (2006). Mapping the hazard of extreme rainfall by peaks over threshold extreme value analysis and spatial regression techniques. Journal of Applied Meteorology and Climatology, 45 (1): 108-124.

Beguería, S., LóPez-Moreno, J.I., Lorente, A., Seeger, M., García-Ruiz, J.M. (2003). Assessing the effect of climate oscillations and land-use changes on streamflow in the Central Spanish Pyrenees. Ambio, 32 (4): 283-286.

Calvo-Cases, A., Harvey, A.M., PayÁ-Serrano, J., AleXander, R.W. (1991). Response of badland surfaces in South East Spain to simulated rainfall. Cuaternario y Geomorfología, 5: 3-14.

Cantón, Y., Domingo, F., SolÉ-Benet, A., Puigdefábregas, J. (2001). Hydrological and erosion response of a badlands system in semiarid SE Spain. Journal of Hydrology, 252 (9): 65-84.

CASTElltoRT, F.X. (1995). Erosió, transport i sedimentació fluvial com a integració dels processos geomorfològics d'una conca. Conca de Cal Rodó, Alt Llobregat. Tesis Doctoral. Universidad de Barcelona, 235 pág., Barcelona.

CERDÀ, A. (1999). Seasonal and spatial variations in infiltration rates in badland surfaces under Mediterranean climatic conditions. Water Resources Research, 35 (1): 319-328.

CERdÀ, A., NAVARro, R. (1997). Procesos de erosión en los badlands alicantinos. Investigaciones geográficas, 17: 99-116.

Clotet, N., Gallart, F. (1986). Sediment yield in a mountainous basin under high Mediterranean climate. Zeitschrift für Geomorphologie, 60: 205-215.

CosAndey, C. (1993). Forêt et écoulements: Rôle de la forêt sur la formation des crues et le bilan d'écoulement annuel. Impact d'une coupe forestière. Rapport sectoriel de recherche du Programme CEE "suivi des conséquences d'une coupe forestière sur les phénomènes hydrologiques, hydrochimiques et d'érosion solide dans un bassinversant de Lozère". 81 pág.

DESCROIX, L., OLIVRY, J.C. (2002). Spatial and temporal factor of erosion by water of black marls in the badlands of the French Southern Alps. Hydrological Sciences Journal, 47 (2): 227-242.

DeSIR, G., MARÍN, C. (2007). Factors controlling the erosion rates in a semi-arid zone (Bardenas Reales, NE Spain). Catena, 71 (1): 31-40.

GALlaRT, F., LATRON, J., REGÜES, D. (1998). Hydrological and sediment processes in the research catchments of Vallcebre (Pyrenees). En: Modelling Erosion by water (Boardman, J. y Favis-Mortolock, D., Eds.). NATO-ASI Series 1-55. Springer, pp. 503-511, Berlín. 
GArcía-Ruiz, J.M., ARnÁEZ, J., White, S., Lorente, A., BEgueríA, S. (2000). Uncertainty assessment in the prediction of extreme rainfall events: an example from the central Spanish Pyrenees. Hydrological Processes, 14: 887-898.

García-Ruiz, J.M., Arnáez, J., Beguería, S., Seeger, M., Martí-Bono, C., Regüés, D., LANA-RENAULT, N., White, S. (2005). Runoff generation in an intensively disturbed, abandoned farmland catchment, Central Spanish Pyrenees. Catena, 59: 79-92.

García-Ruiz, J.M., RegüÉs, D., Alvera, B., LANA-Renault, N., Serrano-Muela, P., Nadal-Romero, E., Navas, A., Latrón, J., Martí-Bono, C., Arnáez, J. (2008). Plant cover, flood generation and sediment transport at catchment scale: a gradient of experimental catchments in the central Pyrenees. Journal of Hydrology, 356 (1-2): 245-260.

GoUdIE, A.S. (Ed.), (2004). Encyplopedia of Geomorphology. 2 vol. Routledge, 1156 pág., Londres.

Hewlett, J.D., HibBerT, A.R. (1967). Factors affecting the response of small watersheds to precipitation in humid areas. En: Forest Hydrology (Sopper, W.E. y Lull, H.W. Eds.). Pergamom, pp. 275-290.

IMESON, A.C., KwAAD, G.J.P., VERSTRATEN, J.M. (1982). The relationship of soil physical and chemical properties to the development of badlands in Morocco. En: Badland Geomorphology and Piping (Bryan, R. y Yair, A. Eds.). Geobooks, pp. 47-70.

JORDAN, J.P. (1992). Identification et modélisation des processus de génération des crues. Application au basin versant de la Haute Mentue. Tesis Doctoral. Ecole Polytechnique Fédérale de Lausanne, 318 pág.

JORDAN, J.P. (1994). Spatial and temporal variability of storm-flow generation processes on a Swiss catchment. Journal of Hydrology, 153: 357-382.

LANA-RENAUlT, N. (2008). Respuesta hidrológica y sedimentológica en una cuenca de montaña media afectada por cambios de cubierta vegetal: la cuenca experimental de Arnás, Pirineo Central. Tesis Doctoral. Universidad de Zaragoza, 314 pág., Zaragoza.

Lana-Renault, N., RegüÉs, D., Martí-Bono, C., Beguería, S., Latron, J., NAdAl, E., SERrano, P., García-Ruiz, J.M. (2007). Temporal variability in the relationships between precipitation, discharge and suspended sediment concentration in a Mediterranean mountain catchment. Nordic Hydrology, 38 (2): 139-150.

LATRON, J. (2003). Estudio del funcionamiento hidrológico en una cuenca mediterránea de montaña (Vallcebre, Pirineos Catalanes). Tesis Doctoral. Universidad de Barcelona, 269 pág., Barcelona.

Latron, J., Gallart, F. (1995). Hydrological response of two nested small Mediterranean basins presenting various degradation states. Phys. Chem. Earth, 20: 369-374. 
Latron, J., Soler, M., Llorens, P., Gallart, F. (2008). Spatial and temporal variability of the hydrological response in a small Mediterranean research catchment (Vallcebre, Eastern Pyrenees). Hydrological Processes, 22 (6): 775-787.

LlOPIS, L.N. (1947). El relieve del Alto Valle del Aragón. Estación de Estudios Pirenaicos del Consejo Superior de Investigaciones Científicas, 90 pág., Zaragoza.

LóPez-Moreno, J.I., Beguería, S., GARCíA-Ruiz, J.M. (2006). Trends in high flows in the Central Spanish Pyrenees: response to climatic factors or to land-use change? Hydrological Science Journal, 51 (6): 1039-1050.

LORENTE, A., GARCíA-RuIZ, J.M., BEGuERÍA, S., ARNÁEZ, J. (2002). Factors explaining the spatial distribution of hillslope debris flows. Mountain Research and Development, 22: 32-39.

Martínez-CARreras, N., Soler, M., Hernández, E., Gallart, F. (2007). Simulating badland erosion with KINEROS2 in a small Mediterranean mountain basin (Vallcebre, Eastern Pyrenees). Catena, 71: 145-154.

Martínez-Mena, M., Williams, A.G., Ternán, J.L., Fitzjohn, C. (1998). Role of antecedent soil water content on aggregates stability in a semi-arid environment. Soil \& Tillage Research, 48: 71-80.

MATHYs, N., RichARD, D., GRESILlON, J.M. (2003). Non-linearity in erosion response of a small mountainous and marly basin: The Laval in the Draix experimental catchments, South East, France. Int. Conf. Hydrology of the Mediterranean and semi-arid regions, Montpellier, France. CD-ROM.

Mathys, N., Klotz, S., Esteves, M., DescroiX, L., Lapetite, J.M. (2005). Runoff and erosion in the Black Marls of the French Alps: Observations and measurements at the plot scale. Catena, 63: 261-281.

NAdAl-Romero, E., RegüÉs, D., Martí-Bono, C., SERrano-Muela, P. (2007). Badlands dynamics in the Central Pyrenees: temporal and spatial patterns of weathering processes. Earth Surfaces Processes and Landforms, 32 (6): 888-904.

Nadal-Romero, E., Latron, J., Lana-Renault, N., Serrano-Muela, P., MartíBONO, C., REGÜÉS, D. (2008a). Temporal variability in hydrological response within a small catchment with badland areas, Central Pyrenees. Hydrological Science Journal, 53 (3).

Nadal-Romero, E., Latron, J., Martí-Bono, C., RegüÉs, D. (2008b). Temporal distribution of suspended sediment transport in a humid Mediterranean badland area: The Araguás catchment, Central Pyrenees. Geomorphology, 97 (3-4): 601-616. DOI: 10.1016/j.geomorph.2007.09.009. 
PARDINI, G. (1996). Evoluzione temporale della microtopografia superficiale, della micromorfologia e della struttura in relazione ai processi di meteorizzazione nelle marne smectitiche di Vallcebre. Tesis Doctoral. Universidad de Barcelona. 409 pág., Barcelona.

REGÜÉS, D. (1995). Meteorización física en relación con los procesos de producción y transporte de sedimentos en un área acarcavada. Tesis Doctoral. Universidad de Barcelona, 302 pág., Barcelona.

REGÜÉs, D., Gallart, F. (2004). Seasonal patterns of runoff and erosion responses to simulated rainfall in a badland area in Mediterranean mountain conditions (Vallcebre, Southeastern Pyrenees). Earth Surface Processes and Landforms, 29: 755-767.

SCOGING, H. (1982). Spatial variations in infiltration, runoff and erosion on hillslopes in semi-arid Spain. En: Badland geomorphology and piping. (Bryan, R. y Yair, A. Eds.). Geobooks, pp. 89-112.

Serrano-Muela, M.P., RegüÉs, D., Latron, J., Marti-Bono, C., Lana-Renault, N., NADAL-ROMERO, E. (2005). Respuesta hidrológica de una cuenca forestal en la montaña media pirenaica: el caso de San Salvador. Cuadernos de Investigación Geográfica, 31: 59-76.

Serrano-Muela, M.P., Lana-Renault, N., Nadal-Romero, E., RegüÉs, D., LAtron, J., MARTi-Bono, C., García-Ruiz, J.M. (2008). Forests and water resources in Mediterranean mountains: The case of the Spanish Pyrenees. Mountain Research and Development, 28 (3-4): 279-285.

SolÉ SABARís, L. (1942). La Canal de Berdún. Primera Reunión de Estudios Geográficos, 7: 271-318. Madrid.

Solé-Benet, A., Calvo, A., Cerdà, A., Lázaro, R., Pini, R., Barbero, J. (1997). Influences of micro-relief patterns and plant cover on runoff related to processes in Badlands from Tabernas (SE Spain). Catena, 31: 28-38.

TORRI, D., RODOLFI, G. (2000). Badlands in changing environments: an introduction. Catena, 40: 119-125. 